\title{
Why I hate the index finger
}

\author{
William L. White
}

\begin{abstract}
Why I Hate the Index Finger" by William L. White, M.D., was originally published in Orthopaedic Review, Volume IX, No. 6 (June 1980) pp. 23-29. "The article is reprinted in HAND courtesy of Quadrant HealthCom, Inc., the publisher of The American Journal of Orthopedics (formerly Orthopaedic Review). A related article can be found at http://dx.doi.org/10.1007/s11552010-9278-4."
\end{abstract}

American Association for Hand Surgery 2010

M. F. Freshwater
Deparment of Surgery,
University of Miami School of Medicine,
9100 South Dadeland Boulevard, Suite 502,
Miami, FL 33156-7815, USA
e-mail: mfelix.freshwater@gmail.com

Precision prehension of the hand involves the thumb, index, and middle fingers. Prehension by these three digits is derived through the median nerve which supplies the extrinsic superficialis muscles, profundus muscles, and the flexor pollicis longus. In addition the same nerve supplies the lumbricals of the index and middle fingers and the positioning muscles of the thumb, the opponens, and the palmar abductor. The median nerve also supplies the tactile surfaces of the three digits.

In contrast to precision prehension, grip strength of the hand is primarily an ulnar nerve function and is obtained through the medial three digits and the thumb. The extrinsic profundus muscles of the ring and little fingers as well as their intrinsic muscles are supplied by the ulnar nerve. The tactile sense of the little finger and the medial half of the ring finger are also of ulnar nerve origin. In grip strength, the adductor of the thumb provides the power of contra force, and it too is supplied by the ulnar nerve.

The middle finger is seen to be the pivotal digit in that it serves the needs of both precision and strength. Normally, it supports the index digit in matters of precision, but if need be it alone can assume the role as the only finger of precision. When its special ability of precision is not required, its great size and strength contribute a considerable portion of the gripping power provided by the medial side of the hand.

\section{A Spotlight Specialist}

As everyone knows, the index finger is a great precision performer. It is a spotlight specialist, and as might be expected, it is generally known as the forefinger. It has unquestionably contributed mightily to man's success on Earth. It has been an implement of precision with which man has expressed himself. It has been the probing finger of curiosity. It has been the digit to show the way, to beckon, and to express anger. But despite these abilities and accomplishments of the index finger, I maintain that this digit has a poor personality. It is clumsy and forever getting in the way. It is lazy at every opportunity, and it is at all times arrogant. In general, the index finger refuses to get involved in anything more vigorous than releasing the arrow, dealing from the bottom, pulling the trigger, or putting the finger on someone.

As for its clumsiness, surveys show that it sustains injury more than any other digit of the hand. Figures will also show that injuries to the index finger are most often of a trivial nature even though the index digit has more time loss from duties than any other two digits of the hand combined. In many instances, injury to the index finger has been due to the combination of ineptness and obstinacy. 
To demonstrate what a poor participant the index finger can be, get a pound of eight or ten penny common nails, a well-balanced hammer, and a heavy log into which you can drive the nails. Start four rows of ten nails, each well spaced, with at least two inches of the nails still to be driven into the wood. The object of this little exercise is to hit the individual nails, solidly, with hard, precise hammer strokes. We will grip the hammer differently in nailing each row. First hold it between the thumb and the index finger and strike each of the ten nails in turn. If you manage three good hits out of ten strokes, you will be about average. Now add your middle finger to the hammer and try again. With this addition, you should be able to get five solid hammer blows out of ten. If you add the ring finger, you should hit eight or nine good ones. When you add your little finger and grip the hammer with your entire hand, you should get ten out of ten good strikes. Oh, I forgot to say the results will depend, of course, upon your being effective with a hammer at the outset.

Now let's reverse the procedure. You cannot really hold the hammer with the little finger and thumb so you have to start with the ring and littler fingers and the thumb. Voila! You should strike nine or ten good blows. Now add the middle finger and feel the strength, comfort, and accuracy improve.

It appears that good, accurate hammering doesn't really require an index finger. In many acts of manual function requiring strength, the hand may function superbly without the index digit. As an example, with both the overlapping and the interlocking grips on a golf club, the object seems to be to get the index digit of the nondominant hand out of the act. Due to the way the club is held, the dominant index finger doesn't grip anyway.

\section{Ashamed of Index Digit}

In spite of its singular ability of precision, it is not difficult to feel ashamed of the index finger. After sustaining an inconsequential injury as a result of its own arrogance, it will refuse to perform regardless of circumstances. Not only does it refuse to function, it interferes with the uninjured parts of the hand engaging in useful activity. It does this by standing in the way, fully extended, inviting disaster.

Rehabilitation of a disabled hand is often thwarted by an obstructing index finger busily overreacting in a hypochondriatic delight to some trivial disability. Rehabilitation of the index is always difficult. Tendon grafts, nerve repairs, and fractures of the index finger seldom work out as well as they do in other digits.

While I believe the index digit should be given every chance for full recovery, I feel that it should be eliminated if it refuses to participate in the function of the hand. A sick index finger greatly compromises the potential of the remaining portions of the hand. The suggestion of amputation, however, is usually met with emotional outcries based on bad propaganda-propaganda which has led to the widely held belief that the index is the most important digit of the human hand. Why else would it be called the forefinger? Why else would it be permitted to point, scratch, pick, and pinch, all of which are uncouth acts? Many people have been misled to believe that the great sensitivity of the index and its remarkable feats of precision mask an enormous hidden strength. These poor misguided people believe loss of the index finger is equal, in fact, to loss of sensitivity, precision, and strength in the hand. I have known a patient's wife to weep at the suggestion of amputation of a stiff, twisted, anesthetic, painful, useless, obstructing index digit on her husband's hand.

\section{Amputation Helpful}

In my experience, amputation of a disabling, index ray through the proximal portion of its metacarpal has seldom failed to provide improved manual function and a great sense of relief unless, of course, the patient happens to be a piano player. As for the lost sensitivity and precision, the middle finger will take over automatically and admirably. If you doubt this, wrap a band-aid around the terminal phalanx of the index digit of your dominant hand. Go about your routine activities. In no time at all there is an excellent chance that your middle finger will have taken over the duties of precision and sensitivity. The index finger will be held fully extended - as much in the way as possible - thus enhancing the chances of injury both to itself and to the remainder of the hand.

Several years ago, I presented my views about a concept which I called an "extensor habitus". This is the tendency for a flexor tendon grafted digit to move into extension when the patient is asked to flex the interphalangeal joints. This usually occurs in digits that have suffered prolonged periods of inability to flex these joints. Extensor habitus occurs most frequently in the index digit. The coordinate ability to flex the index finger thus affected is either lost or is so dominated by the remaining voluntary function of extension that the patient must relearn to flex the involved member. However, in many instances - most of which involved the index - the patient is never able to overcome the protective habit of extension. One of the reasons for the index digit being involved in extensor habitus so often is unquestionably the fact that it has its own individual extensor-the extensor indicis proprius. This fact also accounts for the tendency of the little finger to be plagued with extensor habitus. If extensor habitus persists for a long time in the index digit in spite of all efforts to restore function, then it should be amputated. 
It has been my unfortunate experience to have been a contributor to the development of full blown causalgia involving the upper extremity in four patients. In each case the problem began in the index finger. I have seen other causalgias of the upper limb and been amazed to find that almost all of them began in the index finger. Causalgia most often develops from paresthesia subsequent to nerve injury about the index finger. It is often the result of a small tip amputation resulting in the formation of annoying neuromata. It is my practice to ask patients to traumatize these little neuromas by tapping, squeezing, and pounding the part until the complaints disappear. It takes time and persistence, but it does work.

\section{A Case in Point}

Several years ago, I saw a 32-year-old woman with an injury of her right index finger tip sustained 13 years earlier. During the 13 years before visiting my office she had been employed as a secretary-typist, had married, and had two children. In addition to the two children she had also had seven operations on the tip of the index finger stump in an attempt to relieve her of discomfort. On examination of her finger tip, I was surprised to find it much less sensitive than anticipated. When I asked what she had done with the finger for 13 years, she said "Nothing but bump it."

She could not recall using the finger for any purpose since the time of injury. She kept it fully extended most of the time. I was amazed that she had full flexion and extension at the interphalangeal joints. More than half of her index nail bed had remained, and the nail had grown over the tip of the finger. I felt that 13 years with a useless digit was long enough. I suggested ray resection but insisted that she discuss it with her family first and return in 2 weeks.

Eventually, amputation was carried out; she was happy and relieved of her problem. Speaking objectively, sometimes a potentially useful digit may be sacrificed as in the above instance.

Amputation of the index ray need not result in a major cosmetic disturbance. Removal of a stiff, partially amputated index finger will improve the appearance in most instances. Women with resection of the index ray have been known to play cards all afternoon with other ladies without anyone detecting the digital loss.

\section{Increase Range of Motion}

After amputation of the index ray through the proximal portion of the second metacarpal, interesting things happen to the hand. The carpometacarpal joints of the third, fourth, and fifth rays show an increase in range of motion. Even the relatively fixed third metacarpal moves more extensively at its proximal joint revealing that its rigidity is, in part, derived from the second metacarpal to which it is normally tethered by an intermetacarpal ligament.

With this increase in movement at the carpometacarpal joints, the medial side of the hand becomes more adaptive, conforming, and expansile. Patients with index ray amputations will find their span of grasp has increased considerably. After index ray amputation many men with an average hand size find that they can pick up a basketball with one hand with ease. The loss of the rigidly fixed second metacarpal tends to convert the grip from a three-point torque grip to a three-point grip similar to that of a bone clamp.

It is conceivable that there are some acts of manual function that will be made difficult by amputation of the second ray. Strength of the lifting hand is certainly less since there is loss of supporting bone structure.

Amputation of the index finger proximal to the distal interphalangeal joint results in almost total precision loss which is, in turn, equivalent to functional loss of the digit since it is now only slightly longer than the thumb. Good pinch is thus eliminated. If sensation is impaired, only key pinch with the thumb is possible. These factors interfere with the normal function of the remaining parts of the hand. Amputation of the distal phalanx may have some worth, however, if the individual performs heavy work or if powerful grip is required.

Amputation of the index in the proximal phalanx constitutes a liability to the hand. In most instances such amputations should be converted to ray resections to improve function and comfort. A short index stump is traumatized with each use of the hand particularly with grasping large objects, for the stump is in the region of the bottom of the thumb web. This means that any sizeable object that is grasped will strike the tender stump end.

My indications for resection of the index ray are determined by evaluation of the individual patient in terms of his problem. I do not believe anatomical, physiological, or functional criteria alone should be used in determining who should lose index rays. I feel that this is an extremely personal matter in which the patient must comprehend his problem and provide the final and determining decision for amputation.

\section{Patient Must Be Ally}

If I believe amputation would be helpful in terms of time, investment, sound reason, and early functional return I try to explain to the patient the ways in which his crippled index will complicate his life. I then suggest he go home, 
talk to others about it, and observe his hand at work. I attempt to present the facts objectively. I want the patient to convince himself through his own reason. Then the amputation is something the patient and I mutually undertake for his benefit. When you make the patient your enthusiastic ally in reconstructive surgery, you can get him to fly with six or eight small feathers.

The question may arise as to which index finger is most needed. Obviously, the index finger on the dominant hand should be preserved if at all possible. It is the major contributor of small precision acts. The hardworking nondominant hand lifts, holds, supports, and acts as a vice. It picks up cement blocks, holds lumber, and lifts the shovel. Tools, which are generally much lighter, are used in the dominant hand. Because of these activities the nondominant index finger is often subjected to injuries of greater magnitude. An example are paint gun injuries to the tip of the finger. These most often happen to the nondominant index finger. Frequently this injury occurs when the workman has cleaned the paint gun, then puts his finger over the nozzle tip, and pulls the trigger to see if it is unclogged and working. This usually happens when the paint is diluted with some noxious paint solvent.

\section{Resection Technique}

An annular incision is made about the waist of the proximal phalanx of the index finger. The incision is then carried proximally over the radiodorsal aspect of the index metacarpophalangeal joint and continued along the lateral edge of the second metacarpal to a point approximately $1 / 2$ inch proximal to the second carpometacarpal joint.

Subcutaneous vessels and terminal radial sensory nerves are protected and gently displaced laterally and medially. If injured, these small nerves may result in troublesome neuromata. When the investing fascial plane is encountered, incisions are made on either side of the second metacarpal. These incisions will reveal the first two dorsal interosseous muscles and will expose the second metacarpal throughout its length. Both the extensor indicis proprius and the extensor communis to the index are drawn distally and cut short proximally.

In resection of the index ray it is inadvisable to retain any of the available muscle motors as tendon transfers to the middle finger as this may easily disturb normal function and even produce imbalance. Remember, you cannot make normal more normal because that is abnormal.

After cutting the extensors, the first dorsal interosseous muscle is dissected free from the point of its lateral attachment to the dorsal expansion proximally to the base of the second metacarpal. This will reveal the transverse fibers of the adductor pollicis. Care must be taken not to injure the terminal branch of the deep ulnar motor nerve which penetrates the thumb adductor to reach the first dorsal interosseous muscle. This plump dorsal interosseous muscle will be used to contour and cushion the newly created thumb web space. The second dorsal interosseous muscle and the first volar interosseous muscle should be dissected free of the medial aspect of the second metacarpal.

The deep branch of the radial artery enters the hand at the proximal extreme of the cleft between the first and second metacarpals. It, of course, provides the major blood supply of the deep palmar arch. If it is torn or cut at the cleft, hemorrhage may be difficult to control.

Dissection should now be carried to the volar aspect of the metacarpophalangeal joint where the following structures should be identified, clamped, and divided: the volar digital nerves, the digital arteries, the digital veins, the flexor superficialis, the flexor profundus, and the first lumbrical. It is helpful to put curved clamps on nerves and straights on the other structures.

Now the proximal end of the second metacarpal should be divided taking care not to detach the insertions of the extensor carpi radialis longus dorsally or the flexor carpi radialis anteriorly. The remaining stump of the second metacarpal may be as thin as $1 / 4 \mathrm{inch}$. The periosteum of the metacarpal should not be pushed back; a periosteal spur here could become a painful annoyance. It is unwise to resect the entire second metacarpal for it will result in loss of attachment of the flexor carpi radialis and the extensor carpi radialis longus.

By lifting the proximal end of the second metacarpal the first volar interosseous muscle can be further isolated and removed proximally. The second dorsal interosseous should be left in place to abduct the middle finger. All muscle detachments from the second metacarpal should be done extraperiosteally.

As the dissection of the ray is carried distally the strong intermetacarpal ligament will be encountered on the volar medial aspect at the proximal edge of the metacarpophalangeal joint. It is more anteriorly situated than anticipated. Cut this ligament close to the index and remove the bone ray.

At this point, flex the wrist, pull firmly on the superficialis and cut it short. Draw out the profundus somewhat less forcefully and cut off the lumbrical muscle and divide the profundus tendon. The vascular elements should be dissected back to the palmar flap and there ligated and cut. The radial digital nerve can be gently drawn out in the wound and sharply cut proximally. The medial digital nerve should not be drawn out but dissected free back to the first common volar nerve. The fibers of the medial digital nerve of the index should then be greatly dissected from the lateral digital nerve fibers of the middle finger. When approximately $3 / 4$ inches of the nerve has been dissected free, cut it sharply, leaving a short stump. 
The reflected and retained first dorsal interosseous muscle is now twisted slightly and draped over the second metacarpal stump and thence along the radial aspect of the dorsal interosseous muscle. This is not a muscle transfer so tension is not needed. It should be gently fixed in place with a few small chromic catgut sutures in the fascia. It will pad the bony stump.

The incision should be closed from its distal end to its proximal end. A gentle zig-zag closure should be used in the distal wound, care being taken to retain as much skin as possible. This will add greatly to the cosmetic and functional result. Be careful not to let palmar skin gravitate to the dorsum of the thumb web or dorsal skin end up on the palmar side. These displaced areas of skin are easily recognized and call attention to the amputation. The closure should be accomplished without tension. Usually the skin flap on the thumb side is too long. This can be partially corrected by advancing the medial side. If the wound still shows too much lateral skin, excise a small $\mathrm{V}$ of tissue at its proximal end to permit smooth wound closure.

\section{Summary}

The index digit is indeed a digit of precision. If it incurs even a minor degree of sensory disturbance, its great sensitivity and highly touted precision potentials are often permanently lost unless total recovery occurs.

Obviously, I do not like arrogant disabled index digits and believe they should be removed if they cannot be restored to a functional status. There is no in-between with index fingers.

To me, index fingers portray a hideous personality reflecting conceit and pantywaist attitudes. In essence, they are smart-ass digits we can often do without. If I had to lose a finger and had my choice, I would choose first my nondominant hand index ray and next the other index. I find index digits easy to hate and sometimes hard to love.

Another way to say this is strive for functional restoration of the hand and avoid the pitfalls of your own ego trip in which useless and burdensome parts are saved to the detriment of function. 